\title{
LISTA DE ESPECIES DE PECES DE LA CUENCA DEL RÍO QUEGUAY, RÍO URUGUAY BAJO Sofía Paullier ${ }^{1}$, José Bessonart ${ }^{1}$, Elías Brum ${ }^{2}$, Marcelo Loureiro ${ }^{1^{*}}$
}

\author{
${ }^{1}$ Laboratorio de Zoología de Vertebrados, Departamento de Ecología y Evolución, Facultad de Ciencias, Iguá \\ 4225, Montevideo 11400, Uruguay \\ 2 SNAP-DINAMA. Centro de Visitantes, Paso Andrés Perez, Ruta Nacional 4 km 402, Guichón 60008, \\ Uruguay
}

Autor para correspondencia: mapy @ fcien.edu.uy

\begin{abstract}
RESUMEN
El Río Queguay es uno de los principales afluentes del tramo inferior de la cuenca del Río Uruguay. En la confluencia de sus principales afluentes se forman extensos humedales y montes ribereños, que debido a su diversidad biológica y funcionalidad ecosistémica se han integrado al Sistema Nacional de Áreas Protegidas de Uruguay bajo la denominación "Montes del Queguay". A escala nacional la cuenca queda incluida en dos ecoregiones: la Cuesta Basáltica y la Cuenca Sedimentaria al Oeste. A excepción de un listado sin respaldo en colecciones científicas, no existe información sobre su ictiofauna. Por tanto, el objetivo de este trabajo fue elaborar un listado actualizado de la especies de peces de la cuenca del Río Queguay. Considerando todas las fuentes de infomarción, se registraron un total de 97 especies para la cuenca, correspondientes a 61 géneros, 28 familias y nueve órdenes. La diversidad de especies encontradas fue similar a la del mayor afluente de la ecorregión Uruguay bajo, el Río Negro. Se destacan las principales especies migradoras de la cuenca del Plata, una especie de pez anual endémica de los humedales de la cuenca y el registro de una especie exótica invasora (Cyprinus carpio).
\end{abstract}

Palabras claves: Ictiofauna, Río Uruguay bajo, diversidad, Neotropical

\section{ABSTRACT}

LIST OF FISH SPECIES OF THE QUEGUAY RIVER BASIN, LOWER URUGUAY RIVER. The Queguay river is one of the main tributaries of the lower Uruguay river basin. Large wetlands and riparian forest develop at the confluence of its main affluents and according to their biodiversity and ecosystem functions, they have been included in the National Protected Areas System, under the name "Montes del Queguay". At a national scale the basin is included in two ecoregions: the Basaltic Slope and the Western Sedimentary Basin. Except for an unsupported listing in scientific collections, there is no documented information about its ichthyofauna. Therefore, the objective of this work was to prepare an updated list of the fish species in the Queguay River basin. Considering all sources of information, a total of 97 species were registered for the basin, corresponding to 61 genera, 28 families and nine orders. The diversity of species found was similar to that of the largest tributary of the Lower
Uruguay ecoregion, the Río Negro basin. The main migratory species of the La Plata basin stand out, as well as a species of annual fish endemic to the wetlands of the basin, and the presence of an invasive exotic species (Cyprinus carpio).

Keywords: Ichthyofauna, Lower Uruguay river, diversity, Neotropical

\section{INTRODUCCIÓN}

El Río Uruguay es uno de los principales afluentes de la cuenca del Plata, la quinta en extensión en el planeta. El tramo alto ocurre desde sus nacientes (incluyendo sus principales afluentes, los ríos Pelotas y Canoas), hasta el Salto de Yucumã; el tramo medio, desde este salto hasta el Salto Grande; y el tramo inferior, desde el Salto Grande hasta la desembocadura en el Río de la Plata en Punta Gorda (Zaniboni Filho \& Schulz, 2003). Uno de los principales afluentes del tramo inferior es el río Queguay, cuya cuenca $\left(7860 \mathrm{~km}^{2}\right)$ tiene sus nacientes en la Cuchilla de Haedo. Sus mayores tributarios corren en principio en dirección NE-SW y a medida que confluyen adquieren dirección E-W. En la confluencia del Río Queguay Grande con el Río Queguay Chico se forman extensos humedales y densos montes ribereños, que debido a su diversidad biológica y funcionalidad ecosistémica se han integrado al Sistema Nacional de Áreas Protegidas (SNAP) bajo la denominación "Montes del Queguay" (MVOTMA, 2019) (Fig. 1). Además, esta cuenca drena una de las pocas regiones en Uruguay sobre la que no existen centros industriales, grandes ciudades, o represas en el entorno directo de sus márgenes (Litovsky \& Urruty, 2013).

Desde el punto de vista biogeográfico los tramos inferior y medio de la cuenca del Río Uruguay se consideran como parte de una única ecorregión de organismos dulceacuícolas, el Uruguay bajo (Abell et al. 2008). A nivel nacional, Brazeiro et al. (2015a) resaltan la gran variabilidad de riqueza de especies para esta zona obteniendo valores máximos en todo el litoral oeste y extremo norte del país, destacando a los ríos Uruguay y Cuareim. A esta escala, de acuerdo a Brazeiro et al. (2015b) la cuenca del Río Queguay quedaría incluida en dos ecorregiones: la cuenca alta dentro de la Cuesta Basáltica y la cuenca baja en la Cuenca Sedimentaria del Oeste. 


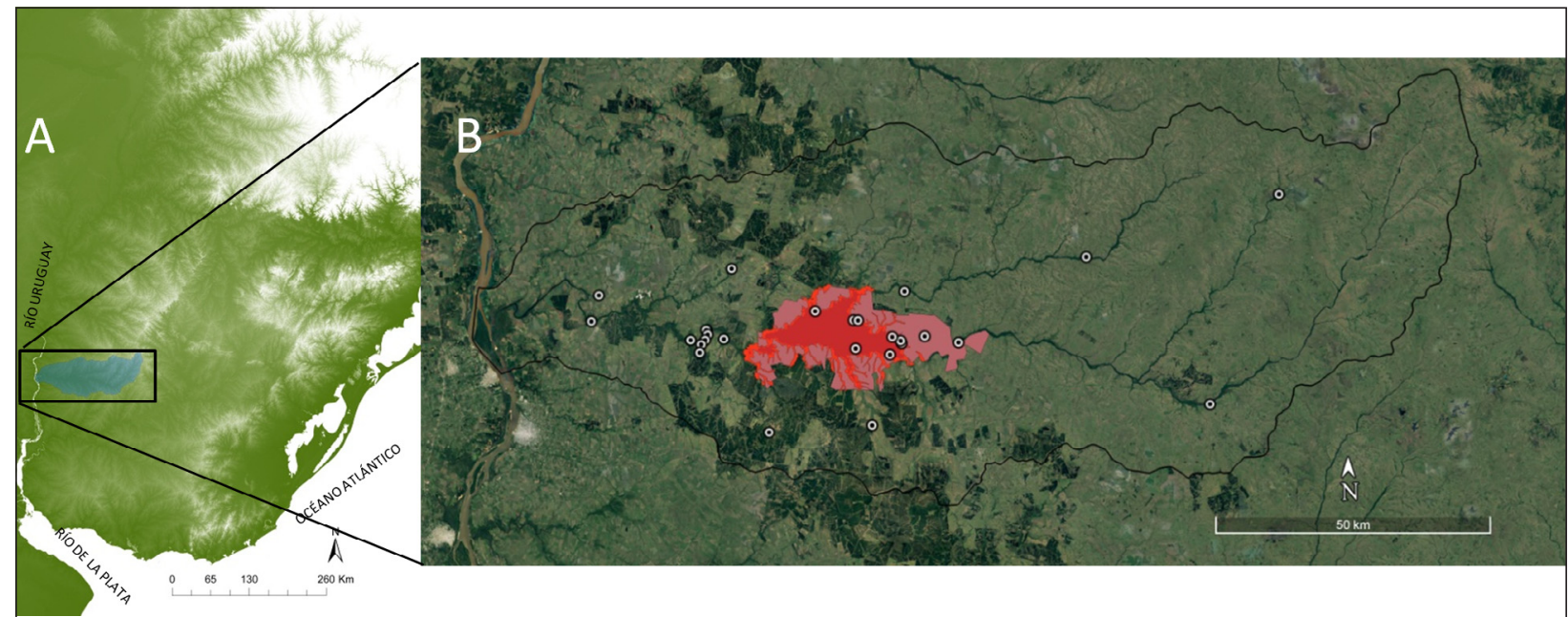

Fig. 1. A. Área sombreada, cuenca del Río Queguay; B. Puntos de registro dentro de la cuenca; área roja: límites del Área Protegida Montes del Queguay; área rosada: límites del área de amortiguación. (MVOTMA, 2019)

Las primeras referencias de ictiofauna para la cuenca del Río Queguay son dos especies de Siluriformes, Scleronema angustirostre (Trichomycteridae) y Trachelyopterus teaguei (Auchenipteridae), descriptas por Garibaldi Devincenzi (Devincenzi \& Teague, 1942), y cuyas localidades típicas se encuentran en este sistema. Sin embargo, existe poca información actualizada sobre la ictiofauna de ésta y otras cuencas de la ecorregión (Serra et al., 2014). Litovsky \& Urruty (2013), presentaron una lista de especies de peces relevadas por pescadores en el Río Queguay Grande, registrando 32 especies. Sin embargo, este listado no está respaldado por material en colecciones científicas, por lo que algunas determinaciones de grupos taxonómicamente complejos no pueden ser verificadas. Por tanto, el objetivo de este trabajo es elaborar un listado actualizado de las especies de peces de la cuenca del Río Queguay.

\section{MATERIALES Y MÉTODOS}

Los ejemplares identificados corresponden a 315 lotes colectados en 26 localidades entre 1951 y 2017 pertenecientes a las colecciones de Facultad de Ciencias (ZVC-P) y Museo Nacional de Historia Natural (MNHN), los cuales abarcan toda la cuenca (Fig. 1). En el caso de tres especies, el registro corresponde solamente a una fotografía tomada in situ por pescadores locales la cual se encuentra en el repositorio electrónico de la colección de peces de la Facultad de Ciencias (ZVC-PF). La identificación taxonómica de los ejemplares se realizó en base a Ghazzi (2008), Rodríguez \& Miquelarena (2008), de Lucena et al. (2013), Serra et al. (2014), Almirón et al.
(2015), Loureiro et al. (2016), Tencatt et al. (2016), Rosso et al. (2018), Serra \& Loureiro (2018), SouzaShibatta et al. (2018) y Turcati et al. (2018). Además, se sumaron al listado las especies mencionadas por Litovsky \& Urruty (2013), cuya identificación consideramos no ambigua (Tabla 1). Para comparar la riqueza de especies encontrada con la de la ecorregión Bajo Uruguay y con cuencas adyacentes se utilizó la densidad de especies siguiendo la fórmula utilizada por Albert et al. (2011):

$$
\mathrm{C}=\mathrm{St} / \mathrm{A}^{\mathrm{b}}
$$

Donde $C$ es la densidad, St el número de especies, $A$ el área de la cuenca y $b$ es el exponente de escalamiento de área por especies. De esta manera se corrige la naturaleza no linear del escalamiento de la relación especies/área, en el cual el número de especies aumenta con el exponente $(b<1.0)$. Este exponente fue obtenido por esos autores de la curva de especies por área de todas las eco-regiones de agua dulce del Neotrópico y toma un valor de 0.3348 .

Las especies se categorizaron de acuerdo a su prioridad de conservación en base a Loureiro et al. (2013), según los siguientes criterios: prioritarias para la conservación (incluye tanto especies amenazadas como aquellas con características que las hacen particulares y justifican especial atención a la hora de planificar estrategias de conservación y desarrollo sustentable en el país), a proteger dentro del SNAP (especies que debido a su distribución restringida o estado de amenaza deberán tener estrategias específicas de conservación en el marco del snap), de uso sustentable (especies de valor medicinal, cultural o económico, incluyendo especies con centro de diversidad en Uruguay, o variedades silvestres de especies domesticadas o cultivadas) (Clavijo et al., 2013). 


\section{RESULTADOS}

Se registraron un total de 97 especies para la cuenca, correspondientes a 61 géneros, 28 familias y nueve órdenes (Tabla 1). El orden Characiformes fue el que presentó una mayor riqueza de especies (47 especies) seguido por Siluriformes (33 especies), Cichliformes (nueve especies), Cyprinodontiformes (tres especies), Gymnotiformes (dos especies), y Clupeiformes, Cypriniformes, Pleuronectiformes y
Synbranchiformes (una especie cada uno)(Fig. 2A). Las familias con más especies fueron Characidae (27 especies), Loricariidae (12 especies) y Cichlidae (nueve especies) (Fig. 2B).

La densidad de especies para la cuenca fue de $4.81 \mathrm{spp} / \mathrm{km}^{2}$. Para la eco-región Cuesta Basáltica se registraron 48 especies, de las cuales seis fueron exclusivas (Astyanax stenohalinus, Odontostilbe sp., Hemiancistrus votouro, Rineloricaria isaaci, Rhamdella longiuscula y Jenynsia onca); mientras

Tabla 1. Especies registradas para la cuenca del Río Queguay. Fuente: origen de la información; SNAP: Anexo correspondiente en la lista de especies prioritarias de SNAP (Loureiro et al., 2013); MQ: Montes del Queguay; CSO: Cuenca Sedimentaria del Oeste; CB: Cuesta Basáltica; P: presencia probable.

Nombre común

Fuente

SNAP

$\mathrm{MQ}$

CSO

CB

Clupeiformes

Clupeidae

Platanichthys platana

Anchoíta

Litovsky \& Urruty, 2013

$\mathrm{A} 1,3$

\section{Cypriniformes}

Cyprinidae

Cyprinus carpio

Carpa

ZVCPF 1

X

X

\section{Characiformes}

\section{Crenuchidae}

Characidium pterostictum

Characidium rachovii

Characidium tenue

Characidium aff. zebra

\section{Erythrinidae}

Hoplias lacerdae

Hoplias argentinensis

\section{Tararira tornasol}

Tararira

ZVCP 13330

ZVCP 13999

Serrasalmidae

Serrasalmus maculatus

Parodontidae

Apareiodon affinis

Prochilodontidae

Prochilodus lineatus

\section{Curimatidae}

Cyphocharax spilotus

Cyphocharax saladensis

Cyphocharax voga

Cyphocharax platanus

Steindachnerina biornata

Steindachnerina brevipinna

\section{Anostomidae}

Megaleporinus obtusidens

Bryconidae

Brycon orbignyanus

Salminus brasiliensis

\section{Acestrorhynchidae}

Acestrorhynchus pantaneiro

Salmón criollo

ZVCP 13425

$\begin{array}{ll}\text { Mariposa } & \text { ZVCP } 14020 \\ \text { Mariposa } & \text { ZVCP } 14028 \\ \text { Mariposa } & \text { ZVCP } 13990 \\ \text { Mariposa } & \text { ZVCP } 13985\end{array}$

$\begin{array}{ll}\text { Piraña } & \text { ZVCP } 14006 \\ \text { Virolo } & \\ & \text { ZVCP } 13389 \\ \text { Sábalo } & \\ & \text { ZVCP14754 } \\ \text { Sabalito } & \text { ZVCP 14008 } \\ \text { Sabalito } & \text { ZVCP 13995 } \\ \text { Sabalito } & \text { ZVCP 14002 } \\ \text { Sabalito } & \text { ZVCP 14494 } \\ \text { Sabalito } & \text { ZVCP 14007 } \\ \text { Sabalito } & \text { ZVCP } 13363\end{array}$

ZVCP 13335

ZVCPF 3

ZVCP 14033

Dorado

$\begin{array}{lll}X & X & X \\ X & X & X \\ X & X & X \\ X & X & \end{array}$

$\begin{array}{clll}A 1,3,5 & P & X & \\ A 1,5 & X & X & X\end{array}$

$X$

$x$

X
$A 1,5$

$\begin{array}{llll}\text { A1,5 } & P & X & X \\ & & & \\ \text { A1,3 } & X & X & X \\ & X & X & \\ & X & X & \\ & X & X & \\ & X & X & X \\ & P & X & \end{array}$

A1,5 $\quad P \quad X$

A1,3,5 $\quad X \quad X$

A1,5 $\quad X \quad X$


Tabla 1. Cont.

Nombre común

\section{Characidae}

Ectrepopterus uruguayensis

Astyanax cf. henseli

Astyanax eigenmanniorum

Astyanax lacustris

Astyanax rutilus

Astyanax stenohalinus

Hyphessobrycon anisitsi

Hyphessobrycon luetkenii

Hyphessobrycon meridionalis

Hyphessobrycon togoi

Hyphessobrycon igneus

Oligosarcus oligolepis

Oligosarcus jenynsii

Charax stenopterus

Cynopotamus argenteus

Heterocheirodon yatai

Cheirodon interruptus

Cheirodon ibicuiensis

Macropsobrycon uruguayanae

Odontostilbe sp.

Pseudocorynopoma doriae

Bryconamericus iheringii

Bryconamericus poi

Bryconamericus stramineus

Diapoma alburnus

Diapoma terofali

Diapoma uruguayensis

\section{Siluriformes}

\section{Trichomycteridae}

Ituglanis australis

Scleronema angustirostre

\section{Callichthydae}

Corydoras longipinnis

Corydoras paleatus

\section{Loricariidae}

Ancistrus taunayi

Hemiancistrus cf. votouro

Hypostomus aspilogaster

Hypostomus commersonni

Hypostomus isbrueckeri

Hypostomus Iuteomaculatus

Hisonotus charrua

Otocinclus arnoldi

Loricariichthys anus

Rineloricaria isaaci

Rineloricaria cf. pareiacantha

Rineloricaria cf. thrissoseps

\section{Doradidae}

Oxydoras kneri

Pterodoras granulosus

\section{Heptapteridae}

Heptaterus mustelinus

Pimelodella australis

Pimelodella gracilis

Mojarra

Mojarra

Mojarra

Mojarra

Mojarra

Mojarra aletuda

Mojarra

Mojarra

Mojarra

Mojarra

Mojarra

Mojarra

Vieja de agua

$\begin{array}{cc}\text { Mojarra } & \text { ZVCP14026 } \\ \text { Mojarra } & \text { ZVCP } 13573 \\ \text { Mojarra } & \text { ZVCP } 14030 \\ \text { Mojarra } & \text { ZVCP } 13361 \\ \text { Mojarra } & \text { ZVCP } 14017 \\ \text { Mojarra } & \text { ZVCP } 13317 \\ \text { Mojarra } & \text { ZVCP } 13964 \\ \text { Mojarra } & \text { ZVCP13963 } \\ \text { Mojarra } & \text { ZVCP 13986 } \\ \text { Mojarra } & \text { ZVCP } 14005 \\ \text { Mojarra } & \text { ZVCP } 13427 \\ \text { Dientudo } & \text { ZVCP } 14032 \\ \text { Dientudo } & \text { ZVCP } 14016 \\ \text { intudo jorobado } & \text { ZVCP } 14015\end{array}$

Dientudo jorobado

Litovsky \& Urruty, 2013

ZVCP 13966

ZVCP 14018

ZVCP 13441

ZVCP 13967

ZVCP 13291

ZVCP 13962

ZVCP 14031

ZVCP 13413

ZVCP 13432

ZVCP13394

ZVCP 13981

ZVCP 14022

\begin{tabular}{|c|c|c|}
\hline $\mathrm{A} 1,4$ & $X$ & $X$ \\
\hline & & $X$ \\
\hline & $X$ & $X$ \\
\hline $\mathrm{A} 1,3$ & $X$ & $X$ \\
\hline & $X$ & $X$ \\
\hline & $X$ & $X$ \\
\hline & $X$ & $X$ \\
\hline & $x$ & $X$ \\
\hline & $X$ & $X$ \\
\hline & $X$ & $x$ \\
\hline & $X$ & $x$ \\
\hline & $X$ & $X$ \\
\hline & $X$ & $X$ \\
\hline
\end{tabular}

A1,3

A1,3

A1,3

A1,3

$\begin{array}{lll}X & X & X \\ X & X & X\end{array}$

Bagre lápiz

Bagre de la arena

ZVCP 13433

ZVCP 14021

Limpiafondos

Limpiafondos

ZVCP 14010

ZVCP 14231

Vieja de agua

Vieja de agua

Vieja de agua

Vieja de agua

Vieja de agua

Vieja de agua

Limpiavidrio

Limpia fondos

Vieja de agua

Vieja de agua

Vieja de agua

Armado chancho

Armado común

Litovsky \& Urruty, 2013

Litovsky \& Urruty, 2013

A1,3,5

A1,3,5
Bagre anguila

Bagre burrito

Bagre burrito
ZVCP 14012
ZVCP 13341
ZVCP 13355
A1

A1,5

ZVCP 14025

ZVCP 13405

ZVCP 13339

Litovsky \& Urruty, 2013

ZVCP 13385

ZVCP 13991

ZVCP 13350

ZVCP 13402

ZVCP 14217

ZVCP 11604

$\begin{array}{cccc}A 1,5 & X & X & X \\ A 1 & X & & X\end{array}$

A1,3,5

A1,5

A1,3,5

A1,3,5

A1,5

$A 1,3,5$

$\begin{array}{lll}X & X & \\ X & X & X\end{array}$

$\begin{array}{lll}X & X & X \\ x & x & x\end{array}$

\section{$X$
$X$}

$X$
$X$

$x$

$\begin{array}{ll}P & X \\ P & X\end{array}$

$\begin{array}{lll} & X & X \\ X & X & \\ P & X & \\ & & X \\ X & X & X \\ X & X & X\end{array}$

Bol. Soc. Zool. Uruguay (2ª época). 2019. Vol. 28 (2): 66-78 ISSN: 0255-4402

$\begin{array}{lll}X & X & X \\ X & X & X\end{array}$


Tabla 1. Cont.

\begin{tabular}{|c|c|c|c|c|c|c|}
\hline & Nombre común & Fuente & SNAP & MQ & CSO & CB \\
\hline Rhamdella longiuscula & Bagre & ZVCP 13395 & & & & $X$ \\
\hline Rhamdia aff. quelen & Bagre negro & ZVCP 14034 & $A 1,5$ & $X$ & $X$ & $X$ \\
\hline $\begin{array}{l}\text { Pimelodidae } \\
\text { Iheringichthys labrosus } \\
\text { Luciopimelodus pati } \\
\text { Parapimelodus valenciennis } \\
\text { Pimelodus maculatus } \\
\text { Pimelodus albicans } \\
\text { Pseudoplatystoma corruscans } \\
\text { Sorubim lima }\end{array}$ & $\begin{array}{l}\text { Bagre trompudo } \\
\text { Patí } \\
\text { Bagre misionero } \\
\text { Bagre amarillo } \\
\text { Bagre blanco } \\
\text { Surubí } \\
\text { Pico de pato }\end{array}$ & $\begin{array}{l}\text { Litovsky \& Urruty, } 2013 \\
\text { Litovsky \& Urruty, } 2013 \\
\text { Litovsky \& Urruty, } 2013 \\
\text { Litovsky \& Urruty, } 2013 \\
\text { Litovsky \& Urruty, } 2013 \\
\text { Litovsky \& Urruty, } 2013 \\
\text { Litovsky \& Urruty, } 2013\end{array}$ & $\begin{array}{c}A 1,5 \\
A 1,5 \\
A 1,5 \\
A 1,5 \\
A 1,3,5 \\
A 1,3,5 \\
A 1,3\end{array}$ & $\mathrm{P}$ & & \\
\hline $\begin{array}{l}\text { Pseudopimelodidae } \\
\text { Microglanis cf. malabarbai }\end{array}$ & languruyú de las piedras & ZVCP 13975 & & $x$ & $x$ & $x$ \\
\hline $\begin{array}{l}\text { Auchenipteridae } \\
\text { Ageneiosus inermis } \\
\text { Trachelyopterus teaguei } \\
\text { Gymnotiformes }\end{array}$ & $\begin{array}{l}\text { Manduví } \\
\text { Torito }\end{array}$ & $\begin{array}{c}\text { Litovsky \& Urruty, } 2013 \\
\text { MHNM } 350\end{array}$ & $\begin{array}{c}\mathrm{A} 1,3,5 \\
\mathrm{~A} 1,3\end{array}$ & $?$ & $?$ & $?$ \\
\hline $\begin{array}{l}\text { Gymnotidae } \\
\text { Gymnotus sp. }\end{array}$ & Pez eléctrico & ZVCP 370 & $\mathrm{~A} 1$ & $\mathrm{P}$ & $?$ & $?$ \\
\hline $\begin{array}{l}\text { Hypopomidae } \\
\text { Brachyhypopomus gauderio } \\
\text { Cichliformes }\end{array}$ & Pez eléctrico & ZVCP 13996 & $\mathrm{~A} 1$ & $x$ & $x$ & \\
\hline $\begin{array}{l}\text { Cichlidae } \\
\text { Australoheros scitulus } \\
\text { Australoheros facetus } \\
\text { Cichlasoma dimerus } \\
\text { Crenicichla lepidota } \\
\text { Crenicichla missioneira } \\
\text { Crenicichla scottii } \\
\text { Gymnogeophagus cf. peliochelynion } \\
\text { Gymnogeophagus terrapurpura } \\
\text { Gymnogeophagus meridionalis } \\
\text { Cyprinodontiformes }\end{array}$ & $\begin{array}{c}\text { Castañeta } \\
\text { Castañeta } \\
\text { Castañeta } \\
\text { Cabeza amarga } \\
\text { Cabeza amarga } \\
\text { Cabeza amarga } \\
\text { Castañeta } \\
\text { Castañeta } \\
\text { Castañeta }\end{array}$ & $\begin{array}{l}\text { ZVCP } 13972 \\
\text { ZVCP14230 } \\
\text { ZVCP } 4159 \\
\text { ZVCP } 14013 \\
\text { ZVCP } 13399 \\
\text { ZVCP } 14014 \\
\text { ZVCP } 13439 \\
\text { ZVCP } 14024 \\
\text { ZVCP } 14009\end{array}$ & $\begin{array}{l}\text { A1,5 } \\
\text { A1,5 } \\
\text { A5 } \\
\text { A5 }\end{array}$ & $\begin{array}{l}X \\
X \\
P \\
X \\
X \\
X \\
X \\
X\end{array}$ & $\begin{array}{l}X \\
X \\
X \\
X \\
X \\
X \\
X \\
X \\
X\end{array}$ & $\begin{array}{l}X \\
X \\
X \\
X \\
X\end{array}$ \\
\hline $\begin{array}{l}\text { Rivulidae } \\
\text { Austrolebias queguay }\end{array}$ & Pez anual & ZVCP 13576 & & $x$ & $x$ & \\
\hline $\begin{array}{l}\text { Poecilidae } \\
\text { Cnesterodon decemmaculatus } \\
\text { Anablepidae }\end{array}$ & Madrecita & ZVCP 14023 & & $x$ & $x$ & $x$ \\
\hline $\begin{array}{l}\text { Jenynsia onca } \\
\text { Pleuronectiformes }\end{array}$ & Overito & ZVCP 13635 & A1,3 & & & $x$ \\
\hline $\begin{array}{l}\text { Achiiridae } \\
\text { Catathyridiumsp. } \\
\text { Synbranchiformes }\end{array}$ & Lenguado & ZVCPF 2 & $A 1,3$ & $\mathrm{P}$ & $\mathrm{P}$ & $x$ \\
\hline $\begin{array}{l}\text { Synbranchidae } \\
\text { Synbranchus marmoratus }\end{array}$ & Anguila & Litovsky \& Urruty, 2013 & $\mathrm{~A} 1$ & & & \\
\hline
\end{tabular}



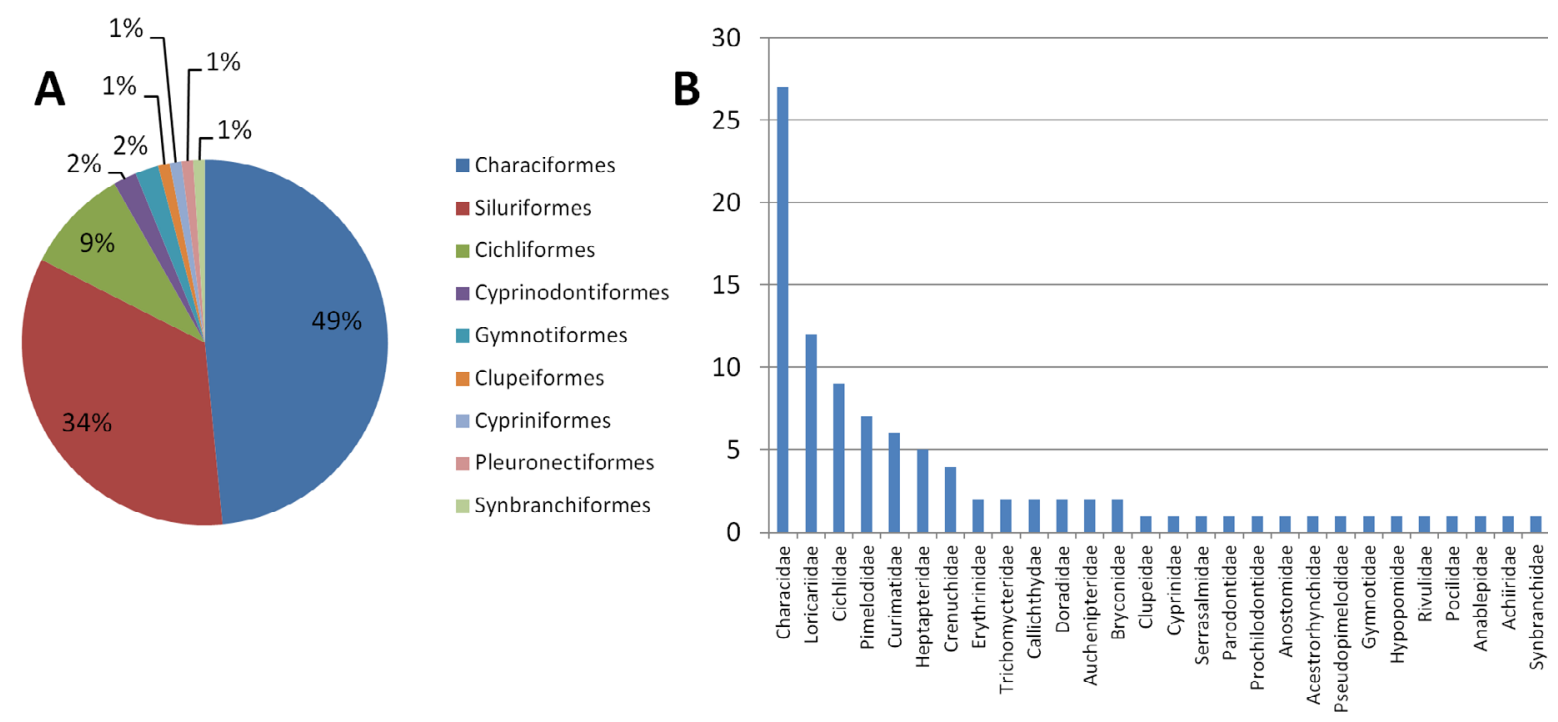

Fig. 2. A. Porcentajes de los diferentes órdenes registrados en la cuenca del Río Queguay; B. Representación de especies por familia registrados en la cuenca del Río Queguay.

que para la eco-región Cuenca Sedimentaria se registraron 74 especies. En el área protegida Montedes del Queguay, que incluye parte de las dos ecoregiones se registraron 76 especies. Se registró una especie endémica para la cuenca del Río Queguay, Austrolebias queguay, que además se encuentra restringida a los humedales del área protegida. Asimismo se registró una especie exótica invasora, la carpa común (Cyprinus carpio).

\section{DISCUSIÓN}

La riqueza de especies reportada en este trabajo para la cuenca del Río Queguay representa el $42 \%$ de las especies reportadas para la eco-región Uruguay bajo (Albert et al., 2011) y sigue el patrón general descrito para la región Neotropical, con el predominio de las especies de los órdenes Characiformes y Siluriformes, y de las familias Characidae, Loricariidae y Cichlidae (Langeani et al., 2005; Cassati et al., 2009; Abrahão et al., 2015). La composición de especies encontrada fue similar a la del mayor afluente de la ecorregión Uruguay bajo, la cuenca del Río Negro (Serra et al., 2014).

La densidad de especies fue alta (4.81 spp/ $\left.\mathrm{km}^{2}\right)$ en comparación con la de la ecorregión Uruguay bajo, la cual fue estimada en $3.6 \mathrm{spp} / \mathrm{km}^{2}$ por Albert et al. (2011), y con la estimada para la cuenca del Río Negro (2.84 spp/ $/ \mathrm{km}^{2}$ ) en base a los datos aportados por Serra et al. (2014). Esto puede estar relacionado a la posición de la cuenca del Río Queguay en el tramo bajo del Río Uruguay. Esta cuenca, con excepción de la relativamente reciente forestación de la parte baja, presenta además grandes extensiones con bajo impacto antropogénico con el uso de la tierra asociado a la ganadería extensiva. Por otra parte, el menor valor obtenido para el Río Negro podría estar asociado a la presencia de las represas hidroeléctricas las cuales han sido postuladas como posibles responsables de algunas extinciones locales (Serra et al. 2014). La alta densidad de especies refleja un buen estado de conservación de la diversidad íctica, y resalta la importancia de la presencia de un área de protección ambiental en la misma.

El menor número de especies presentes en la zona de la Cuesta Basáltica probablemente se debe a que esta zona tiene a los cursos de menor orden dentro de la cuenca, patrón ampliamente documentado para los cuerpos de agua lóticos (Matthews, 1998) y/o al menor número de sitios de colecta registrados. De las cinco especies encontradas exclusivamente en la Cuesta Basáltica, al menos dos están asociadas a ambientes de rápida velocidad de corriente y sustrato rocoso $(H$. votouro y $R$. longiuscula), típicos de esta zona.

Por otra parte, el alto porcentaje del total de especies de la cuenca representadas en el Área Protegida Montes del Queguay (80.8\%), podría ser mayor si se confirma la presencia de varias de las especies mencionadas por Litovsky \& Urruty (2013), de las cuales no se aporta la localización exacta de su 

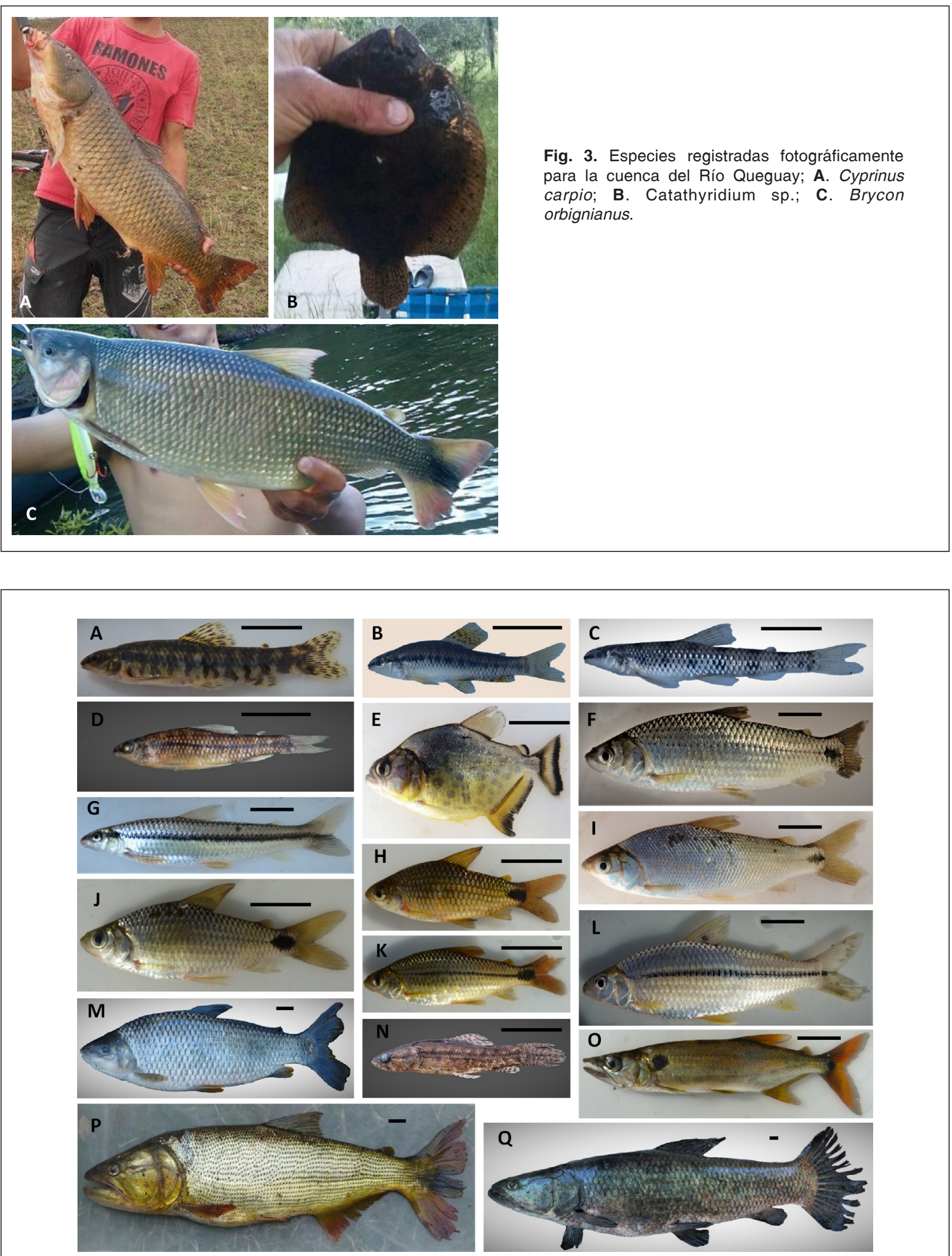

Fig. 4. Especies del orden Characiformes registradas para la cuenca del Río Queguay. A. Characidium pterostictum; B. C. rachovii; C. C. tenue; D. C. aff. zebra; E. Serrasalmus maculatus; F. Cyphocharax voga; G. Apareiodon affinis; H. Cyphocharax saladensis; I. C. platanus; J. C. spilotus; K. Steindachnerina biornata; L. S. brevipinna; M. Megaleporinus obtusidens; N. Hoplias argentinensis; O. Acestrorhynchus pantaneiro; P. Salminus brasiliensis; Q. Hoplias lacerdae. Barra de escala $=1 \mathrm{~cm}$. 


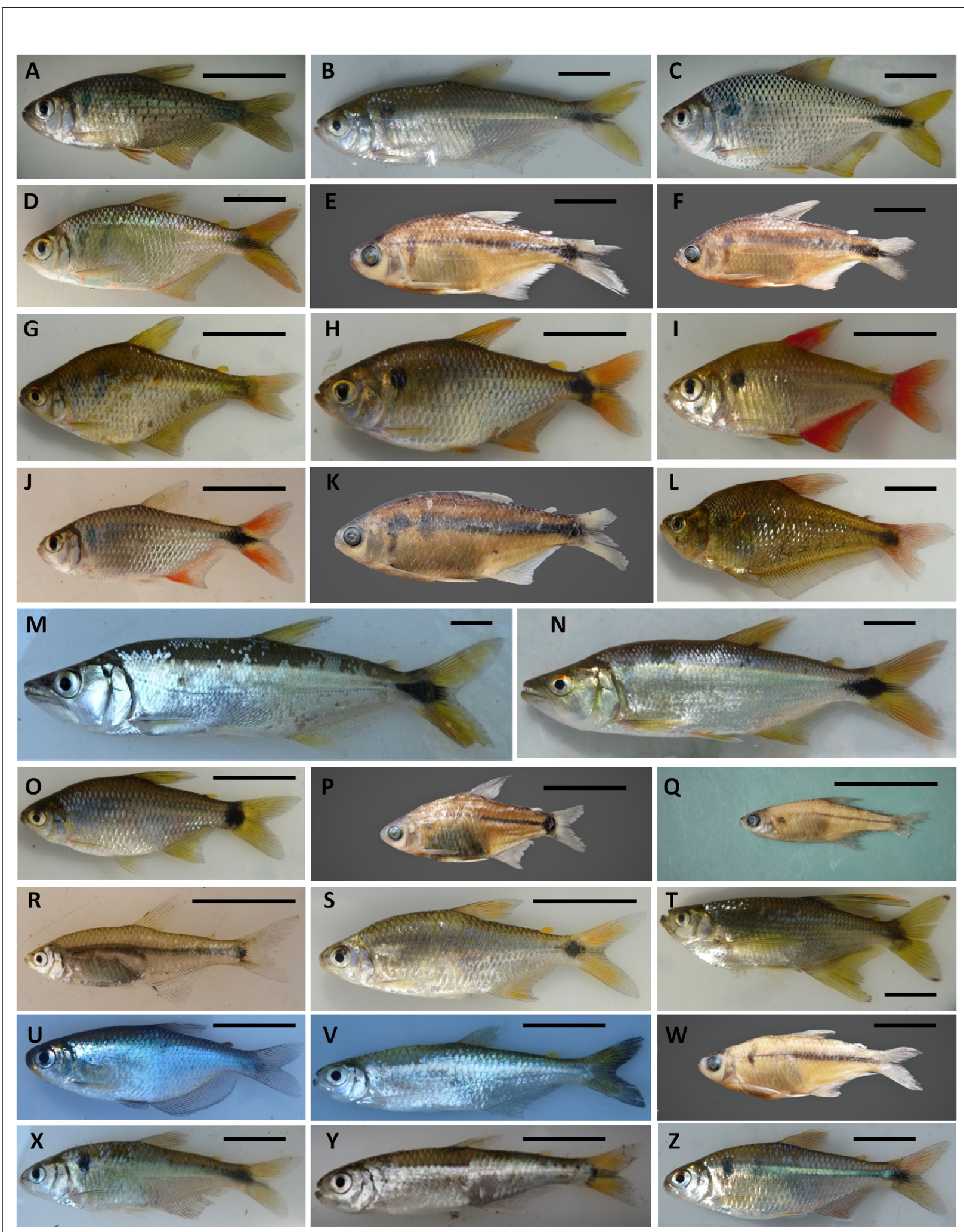

Fig. 5. Especies del orden Characiformes registradas para la cuenca del Río Queguay. A. Ectrepopterus uruguayensis; B. Astyanax rutilus; C. A. lacustris; D. A. eigenmanniorum; E. A. stenohalinus; F. A. aff. henseli; G. Hyphessobrycon meridionalis; H. H. luetkeni; I. H. igneus; J. H. anisitsi; K. H. togoi; L. Charax stenopterus; M. Oligosarcus oligolepis; N. O. jenynsii; O. Cheirodon interruptus; P. C. ibicuhiensis; Q. Heterocheirodon yatai; R. Macropsobrycon uruguayanae; S. Odontostilbe sp.; T. Pseudocorynopoma doriae; U. Diapoma uruguayensis; V. D. alburnus; W. D. terofali; X. Bryconamericus poi; Y. B. stramineus; Z. B. iheringii. Barra de escala $=1 \mathrm{~cm}$. 

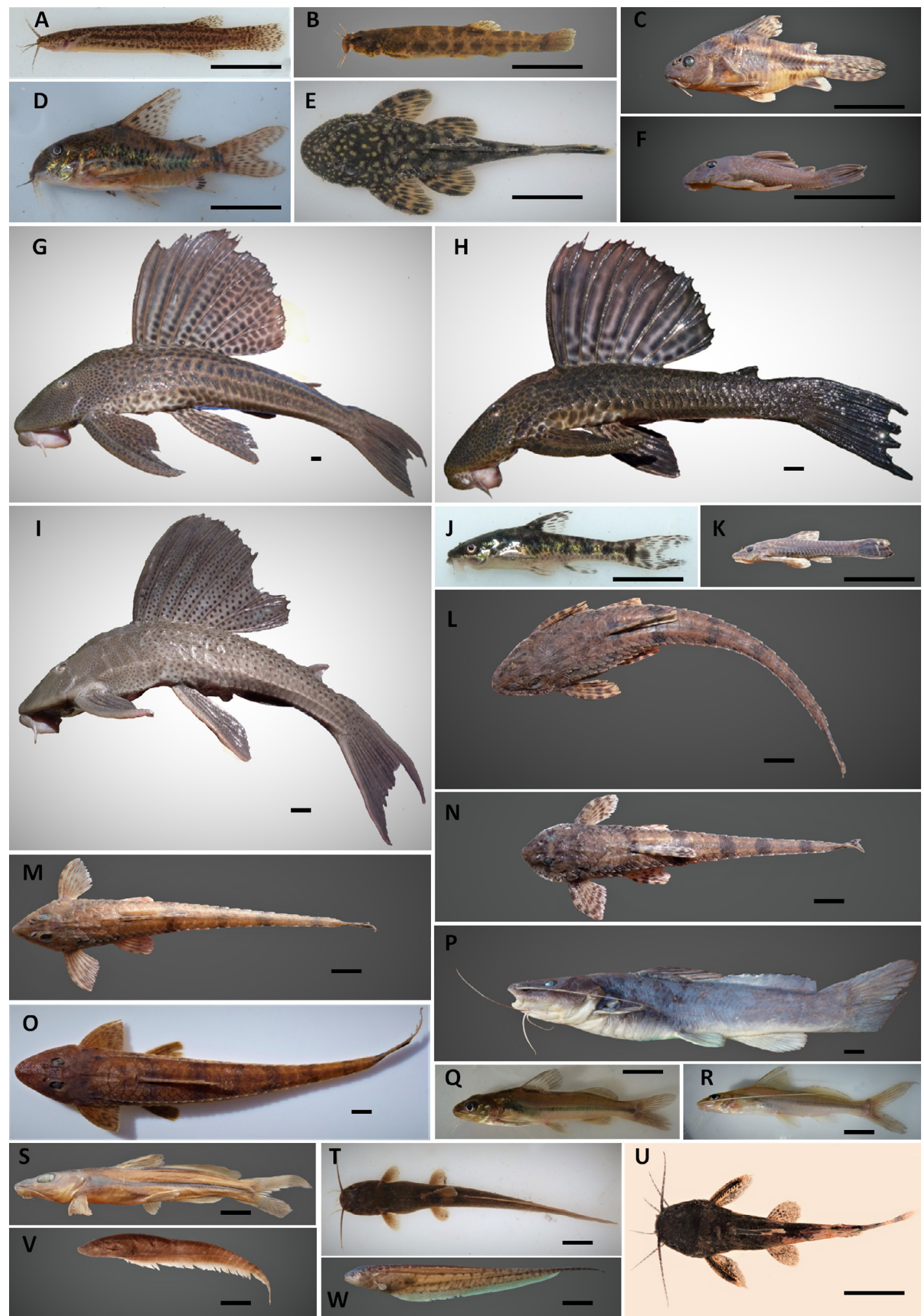

Fig. 6. Especies del orden Siluriformes y Gymnotiformes registradas para la cuenca del Río Queguay. A. Ituglanis australis; B. Scleronema angustirostre; C. Corydoras paleatus; D. C. Iongipinnis; E. Ancistrus taunayi; F. Hemiancistrus cf. votouro; G. Hypostomus aspilogaster, H. H. isbrueckeri; I. H commersonni; J. Otocinclus arnoldi; K. Hisonotus charrua; L. Rinelorcaria isaaci; M. R. cf. pareiacantha; N. R. cf. thrissoseps; O. Loricariichthys anus; P. Rhamdia aff. quelen; Q. Pimelodella australis; R. P. gracilis; S. Rhamdella longiuscula; T. Heptapterus mustelinus; U. Microglanis cf. malabarbai; V. Gymnotus sp.; W. Brachyhypopomus gauderio. Barra de escala $=1 \mathrm{~cm}$. 


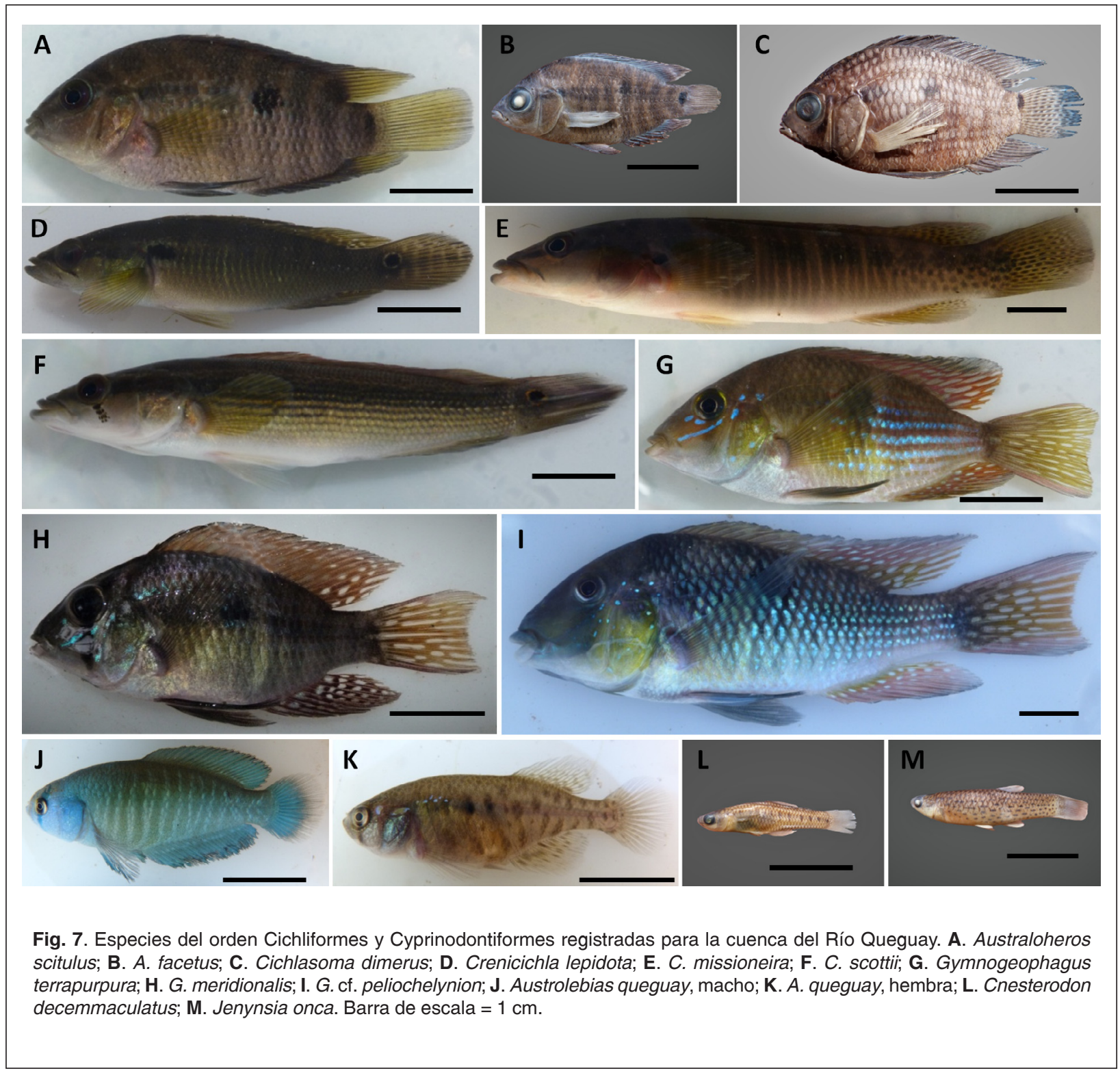

registro. En particular, siete especies de la familia Pimelodidae, de las que no hay ejemplares en las colecciones de referencia. Esta ausencia de los registros formales podría deberse a una alta variabilidad en su presencia dentro de la cuenca o a su distribución restringida a la desembocadura en el Río Uruguay o zonas cercanas a ella. En este sentido destacamos la importancia de confirmar estos registros y de respaldarlos en colecciones científicas nacionales; así como de las restantes especies mencionadas en Litovsky \& Urruty (2013).

Dentro de las especies que se registraron, debido a su distribución restringida en la región, Ectrepopterus uruguayensis (Malabarba et al., 2012), ha sido incluída en la lista de especies prioritarias de SNAP en el Anexo 4, donde se señala la necesidad de su protección en un área de conservación Loureiro et al. (2013). Además, se registraron 24 especies consideradas de importancia como recurso natural (Tabla 1).

Resulta importante la presencia de las principales especies migradoras de la cuenca del Plata. Al respecto, se ha constatado la presencia de adultos de sábalo (Prochilodus lineatus) incluso en sectores de nacientes (M. Loureiro, obs. personal). También cabe destacar el registro del salmón criollo (Brycon orbignyanus), especie considerada amenazada en toda la cuenca del Plata, cuya depencia trófica de frutos y semillas del monte ripario realza la importancia de la conservación de este tipo de vegetación, en la cuenca y en la región (Tonella et al., 2019). La existencia de especies migradoras es de particular interés ya que 
representan un gran aporte en el flujo de energía entre diferentes ecosistemas (Willson \& Halupka, 1995), lo que resalta la importancia del mantenimiento de la conectividad de los afluentes de la cuenca con los tramos bajos y con el Río Uruguay.

Se debe destacar también la presencia de Austrolebias queguay, especie de pez anual endémica de la cuenca y particularmente de los humedales del área protegida (Serra \& Loureiro, 2018). Esto implica una especial atención al momento de establecer los planes de manejo del área, donde, entre otras medidas, se debería asegurar que no se alteren los regímenes hidrológicos que mantienen los humedales donde habitan, especialmente considerando las plantaciones de especies del género Eucalyptus y su influencia sobre la hidrología (Mattos, 2019).

El registro de la especie exótica invasora carpa común (Cyprinus carpio) genera la necesidad de desarrollar un programa de monitoreo en el área que establezca la incidencia de su presencia, con el fin de establecer medidas de prevención de una potencial invasión en esta cuenca. Esta especie ya se ha establecido en nuestro país, especialmente en el Río de la Plata, y hasta el momento solo ha sido registrada de manera esporádica en varios afluentes del Río Uruguay (Zarucki et al. en prensa).

Cabe destacar que algunas especies que aparecen aquí listadas por primera vez en la literatura nacional, no se debe a la ausencia de registros previos, sino a cambios en la nomenclatura. Hoplias argentinensis fue recientemente descrita en base a ejemplares previamente asignados a Hoplias malabaricus (Rosso et al., 2018); Megaleporinus obtusidens fue transferida desde Leporinus obtusidens por Ramirez et al. (2017); Corydoras longipinnis fue descrita en base a ejemplares previamente considerados como $C$. paleatus (Knaak, 2007). En base a una revisión del género, Souza-Shibatta et al. (2018) consideraron que los individuos de Microglanis de la cuenca del Río Uruguay previamente asignados a $M$. cottoides deben ser considerados como Microglanis cf. malabarbai. Por otra parte, la especie de Gymnotus registrada no pudo ser determinada a nivel específico por el estado del material y no necesariamente por tratarse de una especie no descrita previamente. Mientras que Gymnogeophagus cf. peliochelynion fue determinada en base a su parecido a la especie recientemente descrita para la cuenca del Río Arapey (Turcati et al. 2018; W. Serra com. pers.).

Este artículo es la primera publicación sobre la diversidad íctica presente en la cuenca del Río Queguay con respaldo en colecciones científicas, donde se resalta la alta diversidad de especies en comparación con otras cuencas de la región. Destacamos además la posibilidad de utilizar registros fotográficos, que cuando representan una identificación taxonómica inequívoca y se posee datos precisos de su localización, resultan de gran importancia para complementar el conocimiento que se tiene de los componentes de los ecosistemas.

\section{AGRADECIMIENTOS}

Agradecemos a Wiston Albieni y Pablo Garafoni, por su aporte de fotografías de especímenes; a Diego Díaz, Juan Cabrera, Mariana Trillo y Pedro Maisonnave por su colaboración en el trabajo de campo; a Carlín Figari y Gaston Paullier por permitirnos acceder y pernoctar en sus campos; a Francisco Bergós por todo el apoyo logístico en el trabajo; a Wilson Serra por la fotografía de Odontostilbe sp.; y a dos revisores anónimos que mejoraron ostensiblemente la versión final del manuscrito.

\section{REFERENCIAS}

Abell R., Thieme M.L., Revenga C., Bryer M., Kottelat M., Bogutskaya N., Coad B., Mandrak N., Contreras Bladeras S., Bussing W., Stiassny M.L.J., Skelton P., Allen G.R., Unmack P., Naseka A., Sindorf N., Robertson J., Armijo E., Higgins J.V., Heibel T.J., Wikramanayake E., Olson D., López H.L., Reis R.E., Lundberg J.G., Sabaj Pérez M.H. \& Petry P. 2008. Freshwater ecoregions of the World: a new map of biogeographic units for freshwater biodiversity conservation. Bioscience, 58: 403-414.

Abrahão V.P., Claro-García A., Souza-Shibatta L. \& O.A. Shibatta. 2015. Rapid survey of ichthyofauna from rivers and streams of coastal hydrographic regions of Santa Catarina state, Brazil. Check List, 11(5): 1781.

Albert J.S., Petry P. \& R. Reis. 2011. Major Biogeographic and Phylogenetic Patterns. En: Albert J.S. \& Reis R. (Eds.). Historical biogeography of Neotropical freshwater fishes, pp 21-57. University of California Press, Los Ángeles, CA.

Almirón A.E., Casciotta J.R.C., Ciotek L. \& Giorgis P. 2015. Guía de los peces del Parque Nacional Pre-Delta. 2da ed. Administracion de Parques Nacionales, Buenos Aires, Argentina. 300 pp.

Brazeiro A., Achkar M., Bartesaghi L., Ceroni M., Aldabe J., Carreira S., Duarte A., González E., Haretche F., Loureiro M., Martínez-Lanfranco J.A., Maneyro R., Serra W.S. \& Zarucki M. 2015a. Mapeo de la biodiversidad de Uruguay. En:Brazeiro A. (Ed.). Eco-Regiones de Uruguay: Biodiversidad, Presiones y Conservación. Aportes a la Estrategia Nacional de Biodiversidad, pp 22-31. Facultad de Ciencias, CIEDUR, VS-Uruguay, SZU. Montevideo.

Brazeiro A. 2015b. Biodiversidad, conservación y desarrollo en Uruguay. En: Brazeiro A. (Ed.). 
Eco-Regiones de Uruguay: Biodiversidad, Presiones y Conservación. Aportes a la Estrategia Nacional de Biodiversidad, pp 1015. Facultad de Ciencias, CIEDUR, VS-Uruguay, SZU. Montevideo.

Casatti L., Ferreira C.P. \& Langeani F. 2009. A fishbased biotic integrity index for assessment of lowland streams in southeastern Brazil. Hydrobiologia, 623: 173-189.

Clavijo C., Martínez-Lanfranco J.A. \& Soutullo A. 2013. Contribución de una lista de especies prioritarias a la conservación de la biodiversidad en Uruguay: antecedentes, oportunidades y desafíos. En: Soutullo A., Clavijo C. \& MartínezLanfranco J.A. (Eds.). Especies prioritarias para la conservación en Uruguay. Vertebrados, moluscos continentales y plantas vasculares, pp. 17-26. SNAP/DINAMA/MVOTMA y DICYT/ MEC, Montevideo.

de Lucena C.A., Castro J.B. \& Bertaco V.A. 2013. Three new species of Astyanax from drainages of southern Brazil (Characiformes: Characidae). Neotropical Ichthyology, 11(3): 537-552.

Devincenzi G.J. \& Teague G.W. 1942. Ictiofauna del Rio Uruguay medio. Anales del Museo Nacional de Historia Natural de Montevideo (Serie 2), 5(4): 1-100.

Ghazzi M.S. 2008. Nove espécies novas do gênero Rineloricaria (Siluriformes, Loricariidae) do rio Uruguai, do sul do Brasil. Iheringia, Série Zoologia, 98(1): 100-122.

Knaack J. 2007. Beiträge zur Kenntnis der Callichthyidae (Teleostei: Siluriformes). III. Corydoras longipinnis sp. n.-ein neuer Panzerwels aus dem río Dulce in Argentinien (Teleostei: Siluriformes: Callichthyidae). Vertebrate Zoology, 57: 35-55.

Langeani F., Casatti L., Gameiro H.S., Carmo A.B.D. \& Rossa-Feres D.D.C. 2005. Riffle and pool fish communities in a large stream of southeastern Brazil. Neotropical Ichthyology, 3(2): 305-311.

Litovsky M. \& Urruty C. 2013. Memorias del Queguay. Aportes para Plan de Manejo del Área Protegida Montes del Queguay. Contribuciones de los usuarios locales del río Queguay. Grupo creativo de Guichón, Club Queguay Canoas, CEUTA, PPD, GEF, UNDP, ASHOKA, Fondo concursable para la cultura-MEC, Paysandú, Uruguay. 242 pp.

Loureiro M., Zarucki M., Malabarba L.R. \& GonzálezBergonzoni I. 2016. A new species of Gymnogeophagus Miranda Ribeiro from Uruguay (Teleostei: Cichliformes). Neotropical Ichthyology, 14(1): e150082.

Loureiro M., Zarucki M., González I., Vidal N. \& Fabiano G. 2013. Peces continentales. En: Soutullo A., Clavijo C. \& Martínez-Lanfranco J.A. (Eds.).
Especies prioritarias para la conservación en Uruguay. Vertebrados, moluscos continentales y plantas vasculares, pp. 91-112. SNAP/ DINAMA/MVOTMA Y DICYT/MEC, MONTEVIDEO.

Malabarba L.R., Bertaco V.A., Carvalho F.R. \& Litz T.O. 2012. Revalidation of the genus Ectrepopterus Fowler (Teleostei: Characiformes), with the redescription of its type species, E. uruguayensis. Zootaxa, 3204: 4760.

Mattos T.S., Oliveira P.T.S.D., Lucas M.C. \& Wendland E. 2019. Groundwater Recharge Decrease Replacing Pasture by Eucalyptus Plantation. Water, 11(6): 1213.

Matthews W.J. 1998. Patterns in freshwater fish ecology. Chapman and Hall, New York. 756 pp.

MVOTMA. 2019. Ministerio de Vivienda, Ordenamiento Territorial y Medio Ambiente. Información disponible en: http:// www.mvotma.gub.uy/institucional/normativaasociada/decretos/item/10006347-decreto-3432014-creacion-area-protegida-montes-delqueguay. (consultado Julio 2019).

Ramirez J.L., Birindelli J.L. \& Galetti Jr P.M. 2017. A new genus of Anostomidae (Ostariophysi: Characiformes): diversity, phylogeny and biogeography based on cytogenetic, molecular and morphological data. Molecular Phylogenetics and Evolution, 107: 308-323.

Rodriguez M. \& Miquelarena A. 2008. Rineloricaria isaaci (Loricariidae: Loricariinae), a new species of loricariid catfish from the Uruguay River basin. Journal of Fish Biology, 73(7): 1635-1647.

Rosso J.J., González-Castro M., Bogan S., Cardoso Y.P., Mabragaña E., Delpiani M. \& Díaz de Astarloa J.M. 2018. Integrative taxonomy reveals a new species of the Hoplias malabaricus species complex (Teleostei: Erythrinidae). Ichthyological Exploration of Freshwaters, 1076: 1-18.

Serra W. S. \& Loureiro M. 2018. Austrolebias queguay (Cyprinodontiformes, Rivulidae), a new species of annual killifish endemic to the lower Uruguay river basin. Zoosystematics and Evolution, 94: 547-556.

Serra S., Bessonart J., Texeira de Mello F., Duarte A., Malabarba L.R. \& Loureiro M. 2014. Peces del Río Negro. MGAP (Ministerio de Ganadería, Agricultura y Pesca) - DINARA (Dirección Nacional de Recursos Acuaticos), Montevideo. $208 \mathrm{pp}$.

Souza-Shibatta L., Tonini J.F.R., Abrahão V.P., Jarduli L.R., Oliveira C., Malabarba L.R., Sofia S.H. \& Shibatta O.A. 2018. Reappraisal of the systematics of Microglanis cottoides (Siluriformes, Pseudopimelodidae), a catfish from southern Brazil. PloS one, 13(7): e0199963. 
Tencatt L.F.C., Britto, M.R.D. \& Pavanelli C.S. 2016. Revisionary study of the armored catfish Corydoras paleatus (Jenyns, 1842)(Siluriformes: Callichthyidae) over 180 years after its discovery by Darwin, with description of a new species. Neotropical Ichthyology, 14(1): e150089.

Tonella L.H., Dias R.M., Vitorino Jr O.B., Fugi R. \& Agostinho A.A. 2019. Conservation status and bio-ecology of Brycon orbignyanus (Characiformes: Bryconidae), an endemic fish species from the Paraná River basin (Brazil) threatened with extinction. Neotropical Ichthyology, 17(3): e190030.

Turcati A., Serra-Alanis W. S. \& Malabarba L.R. 2018. A new mouth brooder species of Gymnogeophagus with hypertrophied lips (Cichliformes: Cichlidae). Neotropical Ichthyology, 16(4): e180118.

Willson M.F. \& Halupka K.C. 1995. Anadromous Fish as Keystone Species in Vertebrate Communities. Conservation Biology, 9(3): 489-
497.

Zaniboni-Filho E. \& Schulz U.H. 2003. Migratory fishes of the Uruguay River. En: Carolsfeld J., Harvey B., Ross C. \& Baer A. (Eds.). Migratory fishes of South America: Biology, fisheries and conservation status, pp 157-194. The International Bank for Reconstruction and Development/The World Bank, Ottawa, Canada. Zarucki M., Loureiro M., Díaz D., Serra W.S. \& Fabiano G. en prensa. Situación de las especies de peces exóticas e invasoras en Uruguay. En: Brazeiro A., Bresciano D., Brugnoli E. \& Iturburu M. (Eds). Especies exóticas invasoras de Uruguay: distribución, impactos socioambientales y estrategias de gestión, pp XX-XX. CEEI, MVOTMA, UdelaR. Montevideo.

Fecha de Recepción: 23 de agosto de 2019 Fecha de Aceptación: 15 de noviembre de 2019 